\title{
Fusions et défusions municipales : nouvelle ville et service amélioré de lecture publique
}

\author{
Gaston Bernier, Edward A. Collister, Solange Cyr, Françoise Deslauriers, Louise \\ Dion, Rénald Drolet, Nicole Dufresne, Agathe Garon, Jean-Pierre Germain, \\ Marie Goyette, Constance Grégoire, Normand Guérette, Marcel Hudon, \\ Mireille Huneault, André Paradis, Jean Payeur, Jean-Louis Rioux et Françoise \\ Sorieul
}

Volume 50, numéro 2, avril-juin 2004

URI : https://id.erudit.org/iderudit/1030077ar

DOI : https://doi.org/10.7202/1030077ar

Aller au sommaire du numéro

Éditeur(s)

Association pour l'avancement des sciences et des techniques de la

documentation (ASTED)

ISSN

0315-2340 (imprimé)

2291-8949 (numérique)

Découvrir la revue

Citer ce document

Bernier, G., Collister, E. A., Cyr, S., Deslauriers, F., Dion, L., Drolet, R., Dufresne, N., Garon, A., Germain, J.-P., Goyette, M., Grégoire, C., Guérette, N., Hudon, M. Huneault, M., Paradis, A., Payeur, J., Rioux, J.-L. \& Sorieul, F. (2004). Fusions et défusions municipales : nouvelle ville et service amélioré de lecture publique. Documentation et bibliothèques, 50(2), 143-144.

https://doi.org/10.7202/1030077ar

Tous droits réservés (c) Association pour l'avancement des sciences et des techniques de la documentation (ASTED), 2004
Ce document est protégé par la loi sur le droit d'auteur. L'utilisation des services d'Érudit (y compris la reproduction) est assujettie à sa politique d'utilisation que vous pouvez consulter en ligne. 


\section{Fusions et défusions municipales: nouvelle ville et service amélioré de lecture publique ${ }^{1}$}

\section{PAR UN GROUPE DE BIBLIOTHÉCAIRES DE LA RÉGION DE QUÉBEC}

\section{NDLR}

À l'occasion de la consultation référendaire de 2004 au Québec, les citoyens de près d'une centaine de municipalités ont été appelés à décider du statu quo ou du démembrement des villes fusionnées. Un groupe de 18 bibliothécaires de la région de Québec, préoccupé par l'impact de cette décision sur les services de lecture publique et les bibliothèques municipales, a préparé le texte qui suit. Il est à noter qu'il a été écrit avant le référendum. Deux notes infrapaginales l'actualisent en tenant compte des résultats du référendum. Les constats et mises en garde de nos collègues sont particulièrement intéressants si l'on considère les résultats du référendum pour la ville de Québec où seules deux municipalités sur douze ont opté pour la défusion.
1. Des référendums portant sur le maintien ou non de frontières municipales fixées en 2000 par le gouvernement québécois ont eu lieu le dimanche 20 juin 2004 dans un peu moins de 100 villes du territoire et dans les 12 de la «nouvelle ville» de Québec.

Des bibliothécaires de la capitale nationale ont voulu, durant la campagne référendaire, évaluer les effets des démembrements sur les services de lecture publique et ainsi mieux mettre de l'avant les bienfaits des regroupements à cet égard. Le texte fut expédié au Carrefour des lecteurs du journal Le Soleil mais ne fut pas publié, peut-être en raison de sa longueur exceptionnelle pour une telle chronique ou, peut-être, en raison de la publication d'autres lettres sur le même sujet pendant la période.

Le manifeste de la vingtaine de bibliothécaires de la conurbation de Québec, d'une ville ou de l'autre, résidant parfois de l'une et travaillant dans une autre, est reproduit ici dans sa forme originale. Il se veut un témoignage ou une "trace» d'un débat contemporain et d'une vision de services améliorés de lecture publique à Québec et dans les villes intégrées. Reste à savoir si, ex-post, la vision se réalisera (G.B.).

2. Pour mémoire: les citoyens de deux villes (Saint-Augustin-de-Desmaures et L'Ancienne-Lorrette) sur douze se sont prononcés pour le maintien des frontières traditionnelles de leur ville.
L

es résultats ${ }^{2}$ des référendums municipaux à venir auront un impact certain sur les bibliothèques publiques de Québec elle-même et de sa banlieue immédiate. La coopération entre elles, le partage et la rationalisation des ressources documentaires, le catalogage central et l'ouverture à tous les abonnés, quel que soit leur lieu de résidence, constituent les premiers pas vers la réalisation d'un réseau digne des années et des décennies à venir.

Les résultats de la consultation du 20 juin accélèreront ou freineront l'adaptation du réseau à un monde en évolution, à sa croissance, à des services accrus au lectorat, à la composition de collections diversifiées et, surtout, à la disponibilité sur place ou à distance de documents hors de portée d'établissements moins bien pourvus en crédits.

Essentiellement, un réseau plus ample de bibliothèques aura des moyens supplémentaires, lesquels seront en proportion de la taille démographique et économique de la nouvelle Capitale. Jusqu'à maintenant, la pratique généralement observée par l'État québécois repose sur le poids démographique des villes et des villages. Mais la constitution élargie d'entités est actuellement souhaitée et voulue tant par la classe politique que par l'administration publique. Aussi, peut-on supposer que les crédits accordés au cours des prochains exercices budgétaires aux bibliothèques regroupées comporteront une prime à une gouvernance actualisée. Notons que l'avènement de la ville nouvelle a déjà eu comme effet de stimuler le développement des bibliothèques des anciennes villes (projets d'agrandissement ou de construction, augmentation des heures d'ouverture, gratuité, etc.). Par ailleurs, il faut tenir compte du préjugé favorable à la lecture publique et à la culture au sein du conseil municipal de Québec au cours des dernières années. On peut croire que la tendance se maintiendra et que les lecteurs en tireront grand profit.

En effet, les lecteurs profiteront d'une accessibilité sans frein, c'est-à-dire sans tarification et sans barrières municipales. Les frais imposés aux citoyens dans plusieurs des anciennes villes de la CUQ constituaient une taxe déguisée importante pour les familles. Beaucoup ne s'inscrivaient pas. À l'occasion de la fusion, ils furent nombreux à le faire en raison de la gratuité décrétée à ce moment-là. Pour l'avenir, on ne 
sait pas si les villes qui pourraient se détacher du noyau central reviendront à la pratique antérieure. Mais on pourrait s'y attendre en raison de la culture des hommes et des femmes politiques des agglomérations de moindre importance et des revenus toujours trop limités. En somme, la nouvelle ville devrait constituer une garantie que les pratiques d'un autre âge seront oubliées.

Le maintien de Québec dans ses nouvelles frontières et la constitution d'un réseau moderne de bibliothèques de lecture publique permettra une diversification des collections qui n'est pas envisageable si chacune reste sur son quant-à-soi et essaie de vivre en régime plus ou moins autarcique. Des documents vendus à gros prix, qui n'intéressent souvent que des groupes spécialisés restreints, seront négligés pour un lectorat limité alors qu'un bassin de lecteurs plus important en rendrait l'achat possible. De plus, le partage des ressources et la rationalisation des achats à l'intérieur du territoire favoriseront l'acquisition d'un plus grand nombre de titres de documents imprimés ou électroniques. Un réseau plus étendu permettra également de constituer des collections en langues étrangères destinées notamment à tous les néoQuébécois. Les bibliothèques pourraient ainsi contribuer à l'effort collectif visant à favoriser l'accueil des immigrants et à assurer leur maintien à long terme.

Enfin, un réseau de lecture publique plus important (possédant des collections patrimoniales régionales) ouvre des perspectives sur des ententes de coopération ou de collaboration avec les autres établissements documentaires de la Capitale-Nationale. La nouvelle ville est le siège de quelques réseaux de bibliothèques qui auraient intérêt à coopérer avec le réseau de lecture publique. On peut penser aux bibliothèques de l'Université Laval, à celles de la Fonction publique et, peut-être, à celles des cégeps du territoire. $\mathrm{Si}$, à l'avenir, cette perspective se concrétisait, les abonnés des différents réseaux auraient accès à des collections sur presque tous les sujets, à des collections spécialisées, et cela, parfois dans leur voisinage. Bref, après entente, le réseau sera davantage en mesure de profiter des collections des autres bibliothèques de la nouvelle ville et de faire profiter ainsi le lectorat des autres établissements documentaires de ses propres biens culturels.

Le maintien des frontières municipales de Québec établi par le gouvernement Landry, et pour l'instant, discrètement entériné par le gouvernement Charest, signifierait de nombreux bénéfices pour le lectorat des villes tant du centre que de la périphérie. L'évolution et les progrès sont déjà observables en raison de l'impact des nouvelles techniques. Un éclatement de la ville ne serait pas la fin de tout, mais il en découlerait une pause dans les avancées déjà mesurables. À l'inverse, la confirmation des structures mises en place, il y a trois ans, confirmerait le sens de l'évolution et contribuerait à briser les embâcles rencontrées dans la mise en réseau des bibliothèques. $\diamond$

GASTON BERnier, ex-directeur de la Bibliothèque de l'Assemblée nationale

EDWARd A. Collister, autrefois à la Bibliothèque administrative

Solange CYR, bibliothécaire au ministère de l'Éducation

Françoise Deslauriers, retraitée de la Bibliothèque de l'Assemblée nationale

Louise Dion, retraitée de l'Université Laval RÉNALd DROLET, retraité de l'Assemblée nationale

Nicole Dufresne, Bibliothèque de l'Assemblée nationale

Agathe Garon, retraitée de l'Université Laval Jean-Pierre Germain, à titre individuel (Québec)

MARIE GoyetTe, à titre individuel (Beauport)

Constance Grégoire, ex-bibliothécaire de Charlesbourg

NORMAND GUÉRETTE, retraité, autrefois du ministère de l'Énergie et ex-bénévole de la Bibliothèque de Cap-Rouge

MARCEL Hudon, retraité de l'Université Laval et ex-maire deL'Ancienne-Lorrette

Mireille Huneault, bibliothécaire au ministère de l'Éducation (Québec)

ANDRÉ PARADIS, retraité du ministère de la Culture et des Communications

JeAn PAYeur, directeur de l'Institut canadien de Québec (Beauport)

JEAN-LOUIS RIOUX, informaticien et bibliothécaire conseil

FRANÇOISE SORIEUL, à titre individuel

(Québec)

\section{$\begin{array}{llllllllllll}\mathbf{E} & \mathbf{R} & \mathbf{R} & \mathbf{A} & \mathbf{T} & \mathbf{A}\end{array}$}

Dans le volume 49, numéro 4 de Documentation et Bibliothèques, l'auteur du compte rendu $d u$ Dictionnaire biographique des Évêques catholiques du Canada de Jean Leblanc n'apparaît pas. Nous tenons à nous excuser auprès de monsieur Nive Voisine, professeur émérite de l'Université de Laval, de cette malencontreuse erreur.

Dans le volume 50 numéro 1 de Documentation et Bibliothèques, nous mentionnions que la saisie des données avait été faite par Ginette Chandonnet et Louise Marcotte. Il fallait lire que "L'indexation et la saisie des données ont été faites par Ginette Chandonnet et Louise Marcotte». Nous présentons toutes nos excuses aux auteures. 